\title{
Markerless Gait Analysis Vision System for Real-time Gait Monitoring
}

\author{
João André \\ João Lopes \\ Manuel Palermo \\ Diogo Gonçalves \\ Ana Matias \\ Universidade do Minho \\ Universidade do Minho \\ Universidade do Minho \\ Universidade do Minho \\ Hospital de Braga \\ Fátima Pereira \\ Hospital de Braga \\ Eurico Seabra \\ Universidade do Minho \\ João Cerqueira \\ 2CA-Braga \\ Cristina Santos \\ Universidade do Minho
}

\begin{abstract}
On this paper a vision-based contact- and markerless method for gait evaluation is proposed, and validated in different experimental setups against commercial motion capture systems (Vicon) and inertial gait analysis tools (GaitShoes). While the development goal is its integration on the ASBGo Smart Walker platform, only an inexpensive depth camera is required. It is shown to have reasonable results when computing gait metrics in real time, in different experimental setups, from different walker types, vision hardware and walking scenarios. Performance is evaluated through RMSD values for several gait metrics. Results illustrate that the proposed approach can be a valuable non-invasive, contactless and low cost alternative to gait analysis systems used in clinical rehabilitation environments.
\end{abstract}

Index Terms-gait, rehabilitation, smart walker, vision

\section{INTRODUCTION}

In the field of medical assessment and diagnostics, contactless or holistic approaches constitute nowadays desirable alternatives to conventional systems that allow the subject to move freely and comfortably without any kind of restriction. While generally not as precise as their counterparts, occasionally they present the most appropriate solution to the compromise between invasiveness and accuracy. One such case is clinical gait rehabilitation of patients with impaired mobility, where any hindrance to their movement is greatly discouraged by the medical staff [1]. As such, reliable gait analysis systems with minimal invasiveness can have significant relevance in gait rehabilitation. In particular, providing accurate objective gait performance metrics allows the medical team to infer on the patients evolution through several sessions, which in turn can be used to customize/adjust each therapy session to the patient.

Walkers are nowadays a popular gait rehabilitation tool, as they provide a valuable help by increasing the support base, thus providing more stability to the walking process. However, particularly in elderly patients, their usage generally translates to additional cognitive load while navigating the world. In the specific case of post-stroke ataxic patients, rehabilitative success is often achieved through regular assisted therapy sessions using such an assistive walker [2]-[5]. However, elderly and disabled patients often find walking with a walker (or other assistive device) a taxing task and this additional cognitive load can slow their rehabilitation progress [5].

Smart Walkers have been proposed to circumvent this issue, as people who undergo this kind of therapy often present limited mobility or debilitating gait issues, greatly deviating from what is seen as normal locomotion, and therefore present novel challenges when designing automated smart systems to analyze their gait, as success with healthy subjects is not always transferable to these situations. While these devices have been introduced as user-friendly, intuitive solutions to increase the mobility of disabled patients, they still demand additional effort to guide and a steep learning curve is inherent [2], [5]. Moreover, a common complaint is the lack of comfort and requiring the usage of markers and/or inertial and force sensors on lower limbs, prohibiting any daily routine use, on top of often entailing prohibitive financial investment, thus restricting their access by the general population.

As such, cost-effective alternative solutions that are able to autonomously adapt to the patient in a practical, intuitive, non-invasive and generally hassle-free are therefore urgently needed. Even if such solutions are not as precise, an acceptable cost-benefit compromise could help great number of patients in need.

An attempt at a low-cost system is the ASBGo Smart Walker [5](Figure 4), which offers a multi-functional and powerful platform to develop such systems, being equipped with a multiple sensors and actuators, including depth cameras for posture and gait evaluation. Extensive multidisciplinary research has been conducted in recent years [3], [4] with this device, and it has been used with success as a support device for people with mobility issues.

In this paper, a markerless, non-invasive, vision-based algorithm is proposed for real-time gait analysis. It is shown that it is able to provide gait-specific metric measurement with reasonable success through feet tracking using only depth information from popular inexpensive depth cameras. It is extensively validated in 3 distinct scenarios of increasing complexity, comparing it to a precise, robust and well-established commercial motion capture system and an alternative inertial data acquisition system. The authors believe this work illustrates that it is possible to implement gait analysis systems able to balance non-invasiveness with accuracy, which is seen as a 


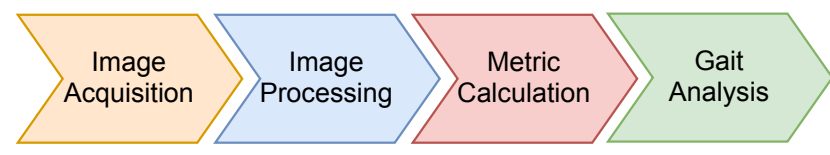

Fig. 1. General top-level flow of the algorithm proposed in this work for feet detection from depth images. On average, Image Acquisition lasts $\sim 1 \mathrm{~ms}$, Image Processing $\sim 2 \mathrm{~ms}$, Gait Parameter Calculation $\sim 3 \mathrm{~ms}$ and Gait Analysis < $1 \mathrm{~ms}$.

real asset within the context of rehabilitation of pathological gait in a clinical setting, and expect this work to provide an base for further development of such solutions.

A brief review of contactless gait analysis systems, along with a detailed description of the vision algorithm implemented in this work, is presented in section II. The experimental setup and the relevant results obtained are discussed in subsection III-B. Lastly, general conclusions and a brief summary of the findings of this work are presented on section IV.

\section{MARKERLESS GAit ANALYSis}

A great number of different approaches have been proposed in the literature for gait analysis. Regarding contactless sensors, they range from ultrasonic [6] sensors to light-based approaches using infrared light (IR) [7] or Laser Range Finders $(L R F)$ modules [8]. A common approach and of particular relevance in the context of this work is the use of depth cameras [2], [9], [10], devices that use structured light (by means of aligned IR emitter matrix and matching sensor) to measure distance.

However, the main limitation with most of these approaches, one that deters their mainstream adoption in rehabilitation procedures, is that they often require the use of markers or wearable accessories, which entails a minimal degree of invasiveness that should nevertheless be avoided when considering patients with limited mobility.

In [2], a depth camera is used to estimate foot position and orientation without the use of any markers. This approach presents a clear evolution over other markerless solutions, with a $25 \%$ decrease in position and orientation errors (roughly $30 \mathrm{~mm}$ RMSD and 21\% RMSD, respectively), while being significantly faster (from $30 \mathrm{~ms}$ per frame, over $15 \mathrm{~s}$ in the literature). The method proposed in this work borrows the same operating principle, but goes beyond tracking feet position and orientation and performs an in-depth gait analysis using several gait indicators useful to the medical staff on a therapy session.

\section{A. Proposed Method}

The main workflow of the vision system proposed in this work is illustrated in Figure 1. Figure 2 depicts several images at different stages of the process. Only the depth frame of the camera is used, with the color image (Figure 2, left) being supplied to subject for visual feedback. After a depth frame is fetched form the camera, it goes through the following stages (Figure 1):

\section{1) Image Processing:}

a) Background Elimination By subtracting a background reference frame ${ }^{1}$ (Figure 2 , center), both the floor and walker's structure are removed. Subsequently, applying a global threshold of $20 \mathrm{~cm}$ from the background reference and cropping the frame to a specific Region of Interest (RoI) enclosing the subject's feet eliminates both the subject's legs and inconsistencies in the surroundings/floor;

b) Feet Segmentation The contours of the feet are then extracted using the Border Following approach supplied by OpenCV, followed by a sequence of morphologic transformations: erosion, closing and dilating. This reduces all relevant pixels to two major blobs corresponding to the subject's feet (Figure 2, right), of which the two closest are assigned to each foot;

2) Metric Calculation Feet position are then used to quantify/identify stance, swing and double support state and duration, step and stride transitions, among other gait metrics used to objectively assess the patient's locomotion; A list of gait metrics and their description is presented in Table I;

3) Gait Analysis Following gait metrics computation, a high-level assessment of the gait characterization is performed in order to provide the patient and assistance/medical staff with objective feedback about the quality of their gait (flagging abnormal situations e.g. excessive distance between feet or a long double support phase).

When considering real time performance, it was observed that the whole image processing routine takes less than $5 \mathrm{~ms}$ (Figure 1 highlights four critical blocks), which allows for use with frame rates up to $120 \mathrm{fps}^{2}$. In this particular work, much lower frame rates were used.

\section{B. Gait Metrics}

Table I presents a detailed list of each metric computed, along with a brief description of each one and how it is computed. Three main groups of metrics are provided: stepspecific, stride-specific and global. There is some overlap over these metrics, as e.g. length, duration and velocity can be found for each.

\section{RESULTS}

\section{A. Experimental Setup}

\footnotetext{
${ }^{1}$ Acquired at the start of the session from frames captured during 5 seconds, while the camera Field of View (FoV) is unobstructed. The minimum depth reading for each pixel is then assumed as background reference value.

${ }^{2}$ On a laptop equipped with a Intel Core i7 CPU @ $2.3 \mathrm{GHz}, 8 \mathrm{~Gb}$ RAM and a AMD Radeon 7970M graphics card.
} 

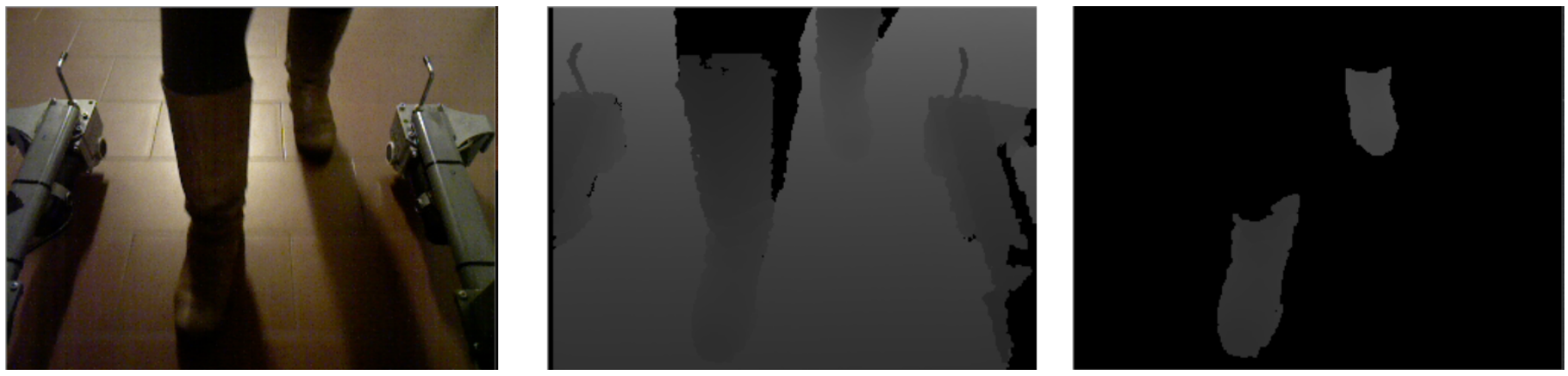

Fig. 2. Frames depicting different aspects of the markerless method proposed in this work. Left Color frame (from the RGB sensor) shown to the user for visual feedback; Center: Depth frame after background subtraction; Right: Left and right feet after segmentation;

TABLE I

GAIT METRICS PROVIDED By THE MARKERLESS GAIT ANALYSIS ALGORITHM

\begin{tabular}{|c|c|c|}
\hline & Name & Description \\
\hline \multirow{6}{*}{ Step } & Foot Angle & Foot orientation at heel-strike $\left(^{o}\right)$ \\
\hline & Width & Lateral distance between feet $(\mathrm{cm})$ \\
\hline & Lengh & Half of the frontal displacement from toe-off to heel-strike ( $\simeq$ torso displacement $)(\mathrm{cm})$ \\
\hline & Duration & Elapsed time from toe off to heel-strike $(s)$ \\
\hline & Velocity & Step Length/Step Duration; Displacement per unit of time $(\mathrm{m} / \mathrm{s})$ \\
\hline & Cadence & Number of steps per unit of time (step/min) \\
\hline \multirow{5}{*}{ Stride } & Lengh & Displacement since heel-strike $(\mathrm{cm})$ \\
\hline & Duration & Elapsed time since heel-strike $(s)$ \\
\hline & Swing time & Elapsed time while the foot/leg is in swing ( $s$ and \% of Stride Duration) \\
\hline & Stance time & Elapsed time while the foot/leg is in stance ( $s$ and \% of Stride Duration) \\
\hline & Double support time & Elapsed time while both feet/legs are in stance ( $s$ and $\%$ of Stride Duration) \\
\hline \multirow{15}{*}{ Global } & Total Distance & Distance traveled during walking session $(m)$ \\
\hline & Total Duration & Total time elapsed during walking session $(\mathrm{min})$ \\
\hline & Average velocity & Total Distance/Total Duration*60 $(\mathrm{m} / \mathrm{s})$ \\
\hline & L/R steps & Number of steps taken with the left/right foot (\#) \\
\hline & L/R cadence & Average number of left/right steps per unit of time (steps/min) \\
\hline & $\mathrm{L} / \mathrm{R}$ distance & Total distance covered by the left/right foot ( $m$ and $\%$ of Total Distance) \\
\hline & L/R walk duration & Cumulative time of all steps take with the left/right foot ( $\mathrm{min}$ and \% of Total Duration) \\
\hline & $\mathrm{L} / \mathrm{R}$ average width & Average step width of steps taken with the left/right foot $(m)$ \\
\hline & $\mathrm{L} / \mathrm{R}$ average length & Average step length of steps taken with the left/right foot $(m)$ \\
\hline & L/R step duration & Average time elapsed by left/right foot steps $(s)$ \\
\hline & L/R stride length & Average length of left/right foot strides $(\mathrm{m})$ \\
\hline & L/R stride duration & Average time elapsed by left/right foot strides $(s)$ \\
\hline & L/R swing duration & Average time elapsed while the left/right foot is in swing ( $s$ and $\%$ of Stride Duration) \\
\hline & $\mathrm{L} / \mathrm{R}$ stance duration & Average time elapsed while the left/right foot is in stance ( $s$ and \% of Stride Duration) \\
\hline & $\mathrm{L} / \mathrm{R}$ double support duration & Average time elapsed while both feet are in stance ( $s$ and $\%$ of Stride Duration) \\
\hline
\end{tabular}

Four different experimental scenarios were considered when evaluating the performance of the vision system proposed in this work, providing different walking contexts such as static and dynamic situations, different velocities, different hardware (depth cameras), allowing a more diverse validation of our approach. Different systems provided a ground truth reference, including a state-of-the-art motion capture system and a set of wearable force/inertial sensing footwear.

1) Motion capture trials: A commercial state-of-the-art vision system was used to establish a ground truth for the proposed vision algorithm.

A Vicon MX 6-camera motion capture system (Vicon Motion Systems, Oxford, UK), provided by the Centro de Computação Gráfica (CCG) at the Universidade do Minho in Guimarães, Portugal, was employed to acquire kinematic data of several key points on multiple subjects. As part of the
Plug-in-Gait module of the Nexus 2.5 software, 16 lower body markers were placed on the test subject, with camera streams being sampled at $120 \mathrm{~Hz}$, and walking trials were conducted using a conventional wheeled walker, equipped with an Asus Xtion depth camera ${ }^{3}$. A total of 10 subjects were asked to follow a straight path for $3.5 \mathrm{~m}$ and then to turn back to their original position. This was repeated 2 times for 3 different (user-defined, non-strict) velocities (slow, normal and fast). Camera streams were sampled at $15 \mathrm{fps}$.

2) Treadmill trials $w /$ healthy subjects: Secondly, a fitness treadmill, available at the Biomedical Robotic Devices (BiRD)

${ }^{3}$ A conventional walker was chosen for these trials in order to be able to be able to track the Vicon markers, which would not be possible with the ASBGo smart walker (obstructing the view). Camera was placed at the same height and orientation as the installation, at a height of $93 \mathrm{~cm}$ and $45 \mathrm{~cm}$ away from the walker wheel. 

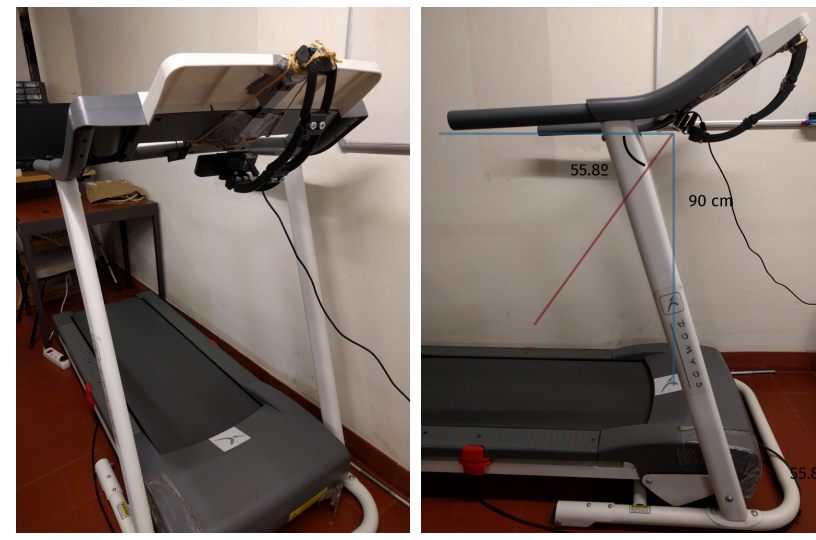

Fig. 3. An Orbbec Astra camera was installed under the treadmill control panel, at a height of $90 \mathrm{~cm}$ and aligned with the start of the walking mat/zone, at an angle of $55.8^{\circ}$ with the walking surface. This setup found empirically to allow both feet to be within the camera's FoV during the entirety of the walking cycle.

laboratory in Guimarães, Portugal, was used for walking trials on a static setting. An Orbbec Astra depth camera, a structured light RGB and depth sensor similar to the Asus Xtion, was installed on an apparatus similar to the one described on the previous section (Figure 3), and sampled at 30 fps. Subjects were asked to walk at pre-defined velocities Table II. In addition to depth camera data, a validation reference was constructed from force and inertial data acquired with a pair of GaitShoes devices [11]. Inertial information was then used to supply foot position and compute gait metrics. Each trial was repeated 3 times for 5 volunteers.

3) Walker trials $w /$ healthy subjects: The third set of trials consisted on a dynamic walk with the ASBGo Smart Walker [3], [4]. The objective was to validate the algorithm on a dynamic setting, closer to daily-life or therapeutic contexts. Both velocities and walking times were different from the static treadmill experiments, though the walking path was kept straight and avoided turning motions.

The ASBGo Smart Walker is equipped with an Asus Xtion depth camera (sampled at $30 \mathrm{fps}$ ) placed at a height of $90 \mathrm{~cm}$ and oriented towards the user's feet with an angle of $55.8^{\circ}$ (Figure 4), providing the same Field-of-View (FoV) as the previous validation setups. 5 volunteers guided the walker on a straight $47 \mathrm{~m}$ corridor at 4 different velocities (Table II ), repeating the trials 3 times for each case. Trials had no fixed duration, instead ending when the walker reached the end of the corridor.

\section{B. Discussion}

For validation purposes, trial repetitions were averaged for both the markerless approach and the reference ground-truth systems, and Root Mean Square Deviation (RMSD) values were computed for each test (velocity) averaging over all trial subjects. Considering the different sample size, velocity values and accuracy of the reference systems, error values are not directly comparable and are only presented to be illustrative
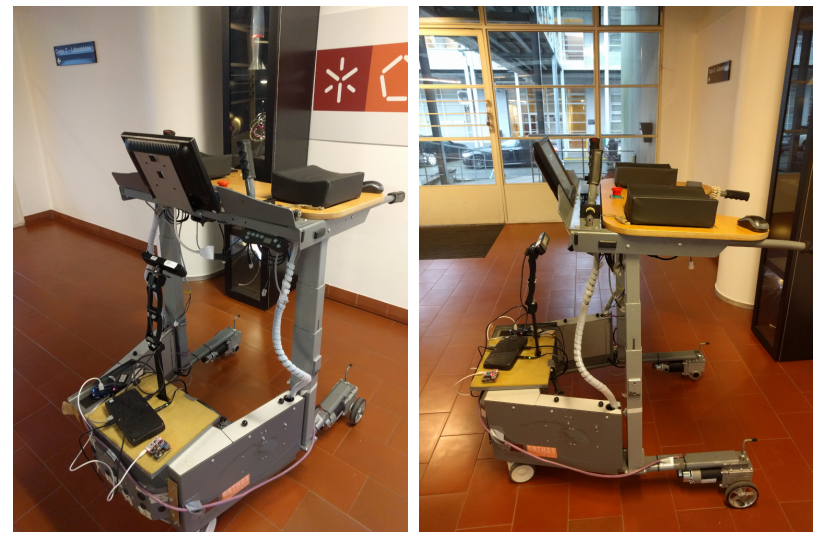

Fig. 4. The ASBGo Smart Walker on a university setting (straight corridor used for validation trials). Camera installation is the same as Figure 3.

of the reliability, versatility and performance of the markerless method. The authors of this work do not believe that a markerless system can hope to match accuracy and precision results than the reference systems adopted, instead aim to show that a markerless system can provide reliable measurements while avoiding some of the pitfalls and shortcoming associated with the use of the former approach. The errors presented in this section originate from the feet detection algorithm and not from metric computation. In fact, the most significant challenge of this work is reliably estimating feet position and velocity. Translating these values to relevant and useful locomotion metrics is assumed to be a straightforward process.

Performance is evaluated based on the difference between measured values with the markerless feet detection and gait analysis algorithm and the reference system adopted in each validation setup, and is presented in Table II. It should be noted, however, that due to the different nature of each metric, it is not possible to find a global error measure. In addition, as described in section II, several metrics are interdependent and thus error sources would have different contributions to each metric. More over, adimensional measures such as percentage (\%) values can be misleading as they depend on the scale of what is being measured. As such, in addition to the global RMSD percentage, metrics were separated into spatial (Step Width, Step Length and Stride Length) and temporal (Step Duration, Stride Duration, Swing Time, Stance Time and Double Support Time), in order to obtain an intuitive quantification of the error associated with the proposed markerless system.

Regarding the reference systems, it should be noted that the Vicon motion capture system and the GaitShoes devices have different accuracy values. While the first is a commercial, wellestablished and proven vision system, the second is a device with known limitations and under current development in a research context (nevertheless, it has been successfully used in gait analysis works [12]). In fact, during the trials with both the treadmill and the ASBGo Smart walker, it was observed that due to electromagnetic interference from both devices, there was a significant sample loss which may have skewed the reference values used for validation purposes. 
TABLE II

GLOBAL, SPATIAL AND TEMPORAL RMSD VALUES MEASURED WITH THE MARKERLESS APPROACH IN EACH OF THE VALIDATION SETUPS DESCRIBED

\begin{tabular}{|c|c|c|c|c|c|c|c|c|c|c|c|}
\hline \multicolumn{4}{|c|}{ Vicon } & \multicolumn{4}{|c|}{ Treadmill } & \multicolumn{4}{|c|}{ Walker } \\
\hline Velocity & Global & Spatial & Temporal & Velocity $(\mathrm{m} / \mathrm{s})$ & Global & Spatial & Temporal & Velocity $(\mathrm{m} / \mathrm{s})$ & $\%$ & Spatial & Temporal \\
\hline $0.4 \mathrm{~m} / \mathrm{s}$ & $35.23 \%$ & $5.19 \mathrm{~cm}$ & $0.234 \mathrm{~s}$ & $0.3 \mathrm{~m} / \mathrm{s}$ & $57.17 \%$ & $15.89 \mathrm{~cm}$ & $1.160 \mathrm{~s}$ & $0.16 \mathrm{~m} / \mathrm{s}$ & $50.06 \%$ & $3.78 \mathrm{~cm}$ & $0.641 \mathrm{~s}$ \\
\hline & & & & $1.0 \mathrm{~m} / \mathrm{s}$ & $30.62 \%$ & $13.52 \mathrm{~cm}$ & $0.075 \mathrm{~s}$ & $0.52 \mathrm{~m} / \mathrm{s}$ & $47.87 \%$ & $8.91 \mathrm{~cm}$ & $0.082 \mathrm{~s}$ \\
\hline
\end{tabular}

Even so, the values achieved illustrate that the markerless approach, while achieving smaller errors in specific metrics/contexts, is generally not as precise and accurate as the reference systems. Still, temporal metrics have a relatively low deviation from the reference values, lower than $0.3 \mathrm{~s}$ in the majority of the situations. Spatial metrics exhibited reasonable accuracy in the trials with Vicon and the ASBGo Smart Walker (5-7 $\mathrm{cm}$ and 3-9 $\mathrm{cm}$, respectively), whereas the Treadmill trials provided slightly higher errors $(13-15 \mathrm{~cm})$.

Considering different walking velocities, there was no consistent trend of increasing or decreasing values with locomotion velocity. At higher walking velocities error magnitude was expected to increase, but that was only observed in the spatial error of the trials with the Smart Walker. In fact, temporal error decreased at greater velocities in all validation trials, as did the spatial error in the trials in the treadmill. Globally, the error percentage value, although unreliable, does suggest higher errors at the lowest velocities.

A great range of RMSD values were observed for each metric, from e.g. low-to-null errors regarding step detection $\left(N_{\text {step }}\right)$ or temporal values such as $T_{\text {step }}$ and $T_{\text {stride }}$, and large outlier values in spatial measurements $L_{\text {step }}$ and $D_{\text {total }}$. This can partially be explained by insufficient device calibration, sporadic feet occlusion or even a inaccurate reference (particularly in the case of the GaitShoes where electromagnetic interference was observed to be significant). Due to the high number of values associated with each metric, only the Step Length, Double Support Time and Average Velocity metrics are considered illustrative of the overall results, and are therefore are further discussed. Figure 5 depicts the variation of the these metrics with the trial velocity for each experimental setup, along with the standard deviation values across subjects. It can be seen that measurements are close to the reference values in most situations, with the exception of visible increased error at the lowest walking velocity in the treadmill trials, in accordance to the values presented in Table II.

As such, the results with Vicon should be considered to represent more robust and reliable than its GaitShoes counter parts, and therefore general conclusions about the performance and accuracy are made based on them.

Summarily, it is the authors' opinion that the error magnitude, while relevant, can be seen as a secondary issue when considering the use of the markerless approach in the rehabilitation of pathological gait, where tracking the evolution the patients takes precedence over accurate and precise mea- surements through foot tracking. As such, the relevant aspect of this work is that it is completely markerless, with only an inexpensive depth camera as hardware requirement.

\section{CONCLUSION}

In this work, a markerless vision-based algorithm for feet detection and gait metric computation was proposed, and validated against different reference systems through walking trials in a myriad of locomotion scenarios. RMSD values were used to evaluate the performance of said system when computing objective gait analysis metrics in real time, and suggest that the proposed approach can provide reliable measurements without the need to place any additional markers on the subject, which is seen as a valuable feature in rehabilitation sessions of patients with limited mobility. Regarding the experimental setup, the reference system used in the treadmill and walker trials was found to have limited reliability, and as such precautions for its use should be taken in future testsy. Still, metrics measured exhibit little deviation from the ones computed with a Vicon motion capture system, which further asserts the valuable contribution of this work. As for future work, tweaking camera installation such as camera position, orientation, extrinsic transformation, frame rate, stereo system, etc. could prove to provide better overall results. Alternatively, more complex tracking algorithms for feet segmentation could improve results in situations such as leg/feet occlusion. In addition, machine learning solutions such as Neural Networks could be employed to streamline feet detection, as they have been shown extensively to be particularly successful in visionbased applications. Finally, in order to better evaluate if the increased error associated with this work is still acceptable for use in rehabilitation treatments, clinical validation trials should be conducted with patients exhibiting pathological gait.

\section{ACKNOWLEDGMENTS}

This work is supported in part by FEDER Funds through COMPETE 2020 - Programa Operacional Competitividade e Internacionalização (POCI) and P2020 with the Reference Project EML under Grant POCI-01-0247-FEDER-033067; and through COMPETE 2020 - Programa Operacional Competitividade e Internacionalização (POCI) with the Reference Project under Grant POCI-01-0145-FEDER-006941. 

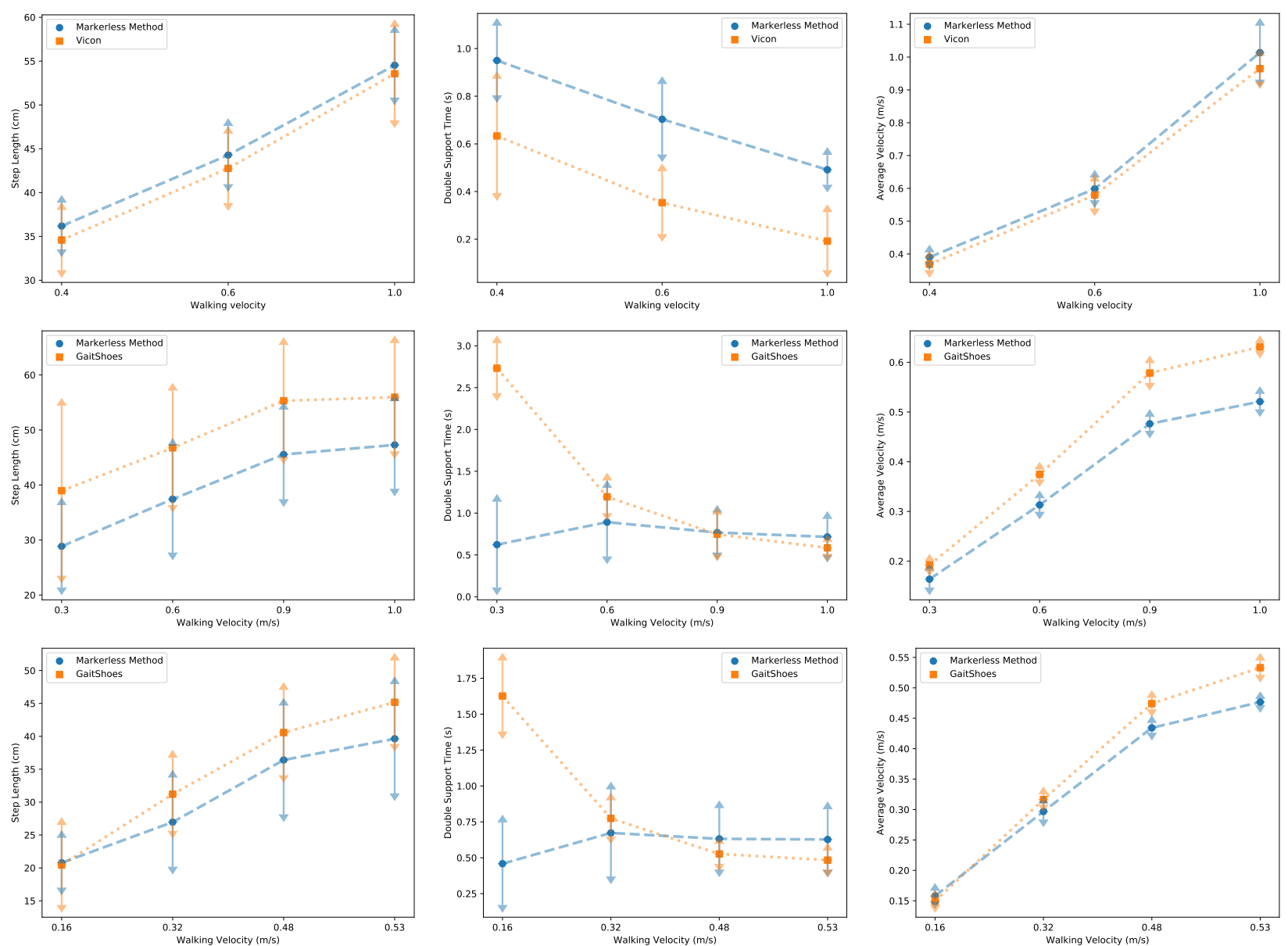

Fig. 5. Average values for 3 different gait metrics in each of the 3 validation setups described in subsection III-A. Values are averaged over all steps of all subjects. Top: Vicon; Middle: Treadmill; Bottom: ASBGo Smart Walker.

\section{REFERENCES}

[1] C. Prakash, R. Kumar, and N. Mittal, "Recent developments in human gait research: parameters, approaches, applications, machine learning techniques, datasets and challenges," Artificial Intelligence Review, vol. 49, no. 1 , pp. 1-40, 2018

[2] P. Solenne, M. Martins, L. Saint-Bauzel, C. Santos, and V. Pasqui, "Fast embedded feet pose estimation based on a depth camera for smart walker," Proceedings - IEEE International Conference on Robotics and Automation, vol. 2015, pp. 4224-4229, 062015.

[3] J. Alves, E. Seabra, I. Caetano, and C. P. Santos, "Overview of the asbgo++ smart walker," in 2017 IEEE 5th Portuguese Meeting on Bioengineering (ENBENG). IEEE, 2017.

[4] A. Pereira, N. F. Ribeiro, and C. P. Santos, "A preliminary strategy for fall prevention in the asbgo smart walker," in 2019 IEEE 6th Portuguese Meeting on Bioengineering (ENBENG). IEEE, feb 2019.

[5] M. Martins, C. P. Santos, A. Frizera, A. Matias, T. Pereira, M. Cotter, and F. Pereira, "Smart walker use for ataxia's rehabilitation: Case study," in 2015 IEEE International Conference on Rehabilitation Robotics (ICORR). IEEE, aug 2015.

[6] S. Paul, A. Banerjee, R. Ghoshal, and D. Tibarewala, "Development of ultrasonic tachography system for gait analysis," International Journal of Biomedical Engineering and Technology, vol. 20, p. 66, 012016.

[7] T.-H. Hwang, J. Reh, A. O. Effenberg, and H. Blume, "Real-time gait analysis using a single head-worn inertial measurement unit," IEEE Transactions on Consumer Electronics, vol. 64, no. 2, pp. 240-248, may 2018.

[8] R. Eguchi, A. Yorozu, and M. Takahashi, "Spatiotemporal and kinetic gait analysis system based on multisensor fusion of laser range sensor and instrumented insoles," in 2019 International Conference on Robotics and Automation (ICRA). IEEE, may 2019.

[9] G. Roy, A. Bhuiya, A. Mukherjee, and S. Bhaumik, "Kinect camera based gait data recording and analysis for assistive robotics-an alternative to goniometer based measurement technique," Procedia Computer Science, vol. 133, pp. 763-771, 2018.

[10] J. Kim, A. Gravunder, C. J. Stanley, and H.-S. Park, "Low-cost implementation of a self-paced treadmill by using a commercial depth sensor," in 2013 35th Annual International Conference of the IEEE Engineering in Medicine and Biology Society (EMBC). IEEE, jul 2013.

[11] J. Figueiredo, C. Ferreira, L. Costa, J. Sepulveda, L. P. Reis, J. C. Moreno, and C. P. Santos, "Instrumented insole system for ambulatory and robotic walking assistance: First advances," in 2017 IEEE International Conference on Autonomous Robot Systems and Competitions (ICARSC). IEEE, apr 2017.

[12] N. F. Ribeiro, J. Figueiredo, and C. P. Santos, "Validation of a wireless communication protocol to monitor human gait using IMUs," in 2017 IEEE 5th Portuguese Meeting on Bioengineering (ENBENG). IEEE, 2017. 